\title{
Modelling the Effects of Disopyramide on Short QT Syndrome Variant 1 in the Human Ventricles
}

\author{
Dominic G Whittaker ${ }^{1}$, Haibo $\mathrm{Ni}^{1}$, Alan P Benson ${ }^{2}$, Jules C Hancox ${ }^{3}$, Henggui Zhang ${ }^{1}$ \\ ${ }^{1}$ University of Manchester, Manchester, UK \\ ${ }^{2}$ University of Leeds, Leeds, UK \\ ${ }^{3}$ University of Bristol, Bristol, UK
}

\begin{abstract}
The short QT syndrome (SQTS) is a recently identified genetic disorder associated with ventricular and/or atrial arrhythmias and increased risk of sudden cardiac death. The SQTS variant 1 (SQT1) N588K mutation to the hERG gene causes a gain-of-function to $I_{K r}$ which shortens the ventricular effective refractory period (ERP), as well as reducing the potency of several drugs which block the hERG channel. This study used computational modelling to assess the effects of disopyramide (DISO), a class 1 a anti-arrhythmic agent, on human ventricular electrophysiology in SQT1.

The O'Hara Rudy dynamic (ORd) model of the human ventricle action potential (AP) was modified to incorporate a Markov chain model of $I_{K r h E R G}$ including formulations for wild type (WT) and SQT1 N588K mutant hERG channels. The blocking effects of DISO on $I_{K r}, I_{N a}$, $I_{C a L}$, and $I_{t o}$ were modelled using $I_{50}$ and Hill coefficient values from the literature. The ability of DISO to prolong the QT interval was evaluated using a $1 D$ model of human ventricular cells with transmural heterogeneities and the corresponding pseudo-ECG.

At a clinically-relevant concentration of $10 \mu \mathrm{M}$ DISO, the action potential duration (APD) at the single cell level was increased significantly through inhibition of mutant SQT1-hERG channels. The corrected QT interval in tissue was prolonged. This study provides further evidence that DISO is a suitable treatment for $h E R G$ mediated SQTS.
\end{abstract}

\section{Introduction}

The short QT syndrome (SQTS) refers to a genetic condition of the heart in which the QT interval on the ECG is abnormally short, which leads to an increased risk of cardiac arrhythmias and sudden cardiac death (SCD) [1]. The first identified form of the SQTS (SQT1) was found to be caused by a missense mutation (N588K) to the human Ether-à-go-go-Related Gene (hERG), which encodes the $\alpha$ subunit of the cardiac rapid delayed rectifier potassium current, $I_{\mathrm{Kr}}$ [2]. The N588K-hERG channel mutation causes a gain-of-function to $I_{\mathrm{Kr}}$ through severely impaired inactivation over the physiological range of membrane potentials [3]. In a prior computational study from our group, this was shown to profoundly reduce ventricular effective refractory period (ERP), decreasing the minimal substrate size necessary to sustain re-entry and increasing the lifespan of re-entrant excitation waves [4]. Whereas multiple proarrhythmogenic effects of SQT1 in the human ventricles have been extensively characterised, less is known about the pharmacology of SQT1.

The current treatment for SQTS patients who show inducible ventricular tachycardia/fibrillation during programmed electrical stimulation is an implantable cardioverter-defibrillator (ICD) device [5]. However, such devices have a well-documented propensity to oversense T-waves (which often appear tall and peaked in SQTS patients) leading to erroneous identification of a tachyarrhythmic event, and are not particularly suitable for paediatric patients [6], necessitating the investigation of alternative, pharmacological approaches.

An in vitro study by McPate et al. [7] used whole-cell patch clamp measurements of $I_{\mathrm{Kr} h \mathrm{R} \text { RG }}$ in Chinese hamster ovary cells at $37^{\circ} \mathrm{C}$ to evaluate the blocking potency of the class 1a anti-arrhythmic drug disopyramide (DISO), where it was seen that N588K mutant hERG channels caused only a modest 1.5 -fold increase in the $\mathrm{IC}_{50}$ (half maximal inhibitory concentration), less than the 3.5-fold increase seen in quinidine, a commonly used drug in the SQT1 setting [8]. The efficacy of DISO in SQT1 in vitro suggests that the drug does not require block of the inactivated state in order to exert its inhibitory effect.

A pilot study showed that oral DISO restored both the QT interval and ventricular ERP in SQT1 patients in vivo, highlighting its potential use as an alternative to quinidine [9]. Here, an in silico approach was taken to gain mechanistic understanding into the action of DISO on human ventricular electrophysiology and consequent effects on QT interval prolongation. 


\section{Methods}

\subsection{Model development}

The O'Hara Rudy dynamic (ORd) model of the human ventricular action potential (AP) [10] was modified to include a previously-described Markov chain formulation of wild type (WT) and N588K mutant $I_{\mathrm{Kr} / \mathrm{K} \text { RGG, with }}$ kinetic changes accounting for impaired inactivation during the AP [11]. The gating scheme of the Markov chain model of the $h E R G$ channel is shown in Figure 1.

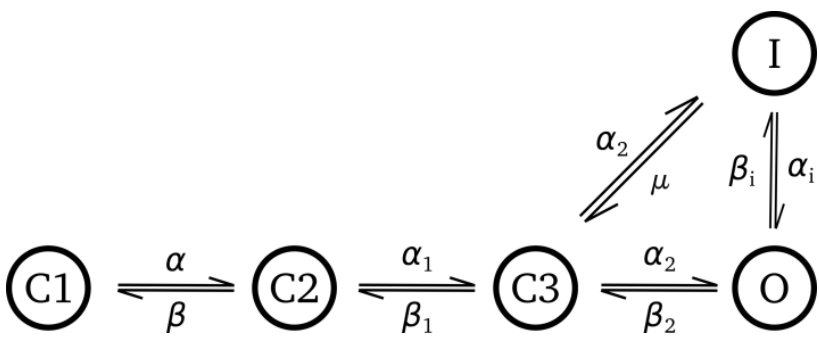

Figure 1: A schematic diagram of the Markov chain model of $I_{\mathrm{Kr} \text { KERG }}$ used in this study. C1-C3 corresponds to closed states, $\mathrm{I}$ is an inactivated state, and $\mathrm{O}$ is the open channel conducting state. Microscopic reversibility is ensured by setting $\mu=\left(\alpha_{\mathrm{i}} \cdot \beta_{2} / \beta \mathrm{i}\right)$.

The SQT1 phenotype was described using a 1:1 ratio of WT to mutant hERG channels, as the N588K mutation is expressed heterozygously in vivo. The conductance of $I_{\mathrm{Kr}}$ was chosen based on the APD prolongation observed under experimental $I_{\mathrm{Kr}}$ block in the human ventricles [12], and the conductances of $I_{\mathrm{Ks}}$ and $I_{\mathrm{K} 1}$ adjusted accordingly. An $I_{\mathrm{Kr}}$ scaling factor ratio of 1.5:1 in epicardial (EPI) to endocardial (ENDO) cells, respectively, was applied as suggested in the study of Adeniran et al. [4], in order to generate a tall, symmetrical T-wave in the pseudo-ECG of the SQT1 phenotype, as seen clinically [2].

The steady-state effects of the class 1a drug DISO on $I_{\mathrm{Kr}}, I_{\mathrm{Na}}, I_{\mathrm{CaL}}$, and $I_{\mathrm{to}}$ were described using $\mathrm{IC}_{50}$ and $n H$ (Hill coefficient) values taken from the literature [7], [13], [14]. The fractional block of each ionic current affected by DISO was modelled using simple pore block theory [15], which postulates that the conductance $g_{i}$ of an ionic current type $i$ is modified in a concentration-dependent manner such that

$$
g_{i}=g_{\text {control }, i} \frac{1}{1+\left(\left[\left[_{50}\right]_{i} /[\text { DISO }]\right)^{n H}\right.},
$$

where [DISO] is the concentration of DISO and all other parameters retain their previous definitions. The blocking potency of DISO on WT/N588K $I_{\mathrm{Kr} h \mathrm{R} G}, I_{\mathrm{Na}}, I_{\mathrm{CaL}}$, and $I_{\mathrm{to}}$ currents from a concentration of $0.1 \mu \mathrm{M}$ to $1000 \mu \mathrm{M}$ is shown in Figure 2.

\subsection{Tissue simulations}

Propagation of APs in tissue was described using the monodomain equation

$$
\partial V_{\mathrm{m}} / \partial t=\nabla \cdot \boldsymbol{D}\left(\nabla V_{\mathrm{m}}\right)-I_{\text {ion }} / C_{\mathrm{m}},
$$

where $V_{\mathrm{m}}$ is transmembrane potential, $\boldsymbol{D}$ is the diffusion coefficient tensor, $I_{\text {ion }}$ is the total ionic current, and $C_{\mathrm{m}}$ is the membrane capacitance. Eq (2) was discretised in space using the finite difference method and a spatial step of $0.15 \mathrm{~mm}$.

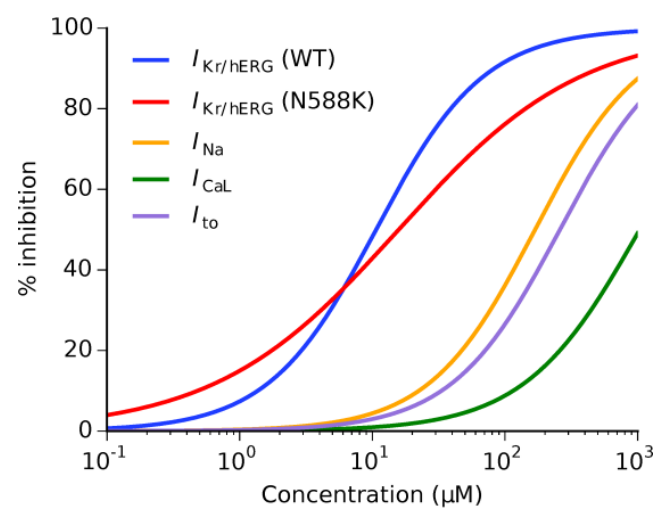

Figure 2: Dose-dependent effects of disopyramide (DISO) on $I_{\mathrm{Kr}}$ (WT and N588K mutant hERG channels shown separately), $I_{\mathrm{Na}}, I_{\mathrm{CaL}}$, and $I_{\mathrm{to}}$.

The 1D transmural model of human ventricle had a size of $15 \mathrm{~mm}$, comprising 25 ENDO, 35 MCELL, and 40 EPI cells, with isotropic diffusion except for a 5-fold decrease in $\boldsymbol{D}$ at the MCELL:EPI border, as described in [4]. A stimulus was applied at the endocardial surface, eliciting a plane wave which propagated transmurally towards the epicardial surface. The pseudo-ECG was calculated using the method of Gima and Rudy [16], i.e.

$$
\Phi\left(x^{\prime}\right)=\frac{a^{2}}{4} \int\left(-\nabla V_{\mathrm{m}}\right) \cdot\left[\nabla \frac{1}{r}\right] d x,
$$

where $\Phi$ is a unipolar potential generated by the $1 \mathrm{D}$ transmural fibre, $a$ is the radius of the fibre, and $r$ is the distance from a source point $x$ to the field point $x$, recorded at a site located $2 \mathrm{~cm}$ away from the epicardial end of the strand. The QT interval was corrected according to Framingham's QT correction formula.

\section{Results}

\subsection{Single cell investigations}

At the single cell level, all concentrations of DISO simulated ( $5 \mu \mathrm{M}, 10 \mu \mathrm{M}, 20 \mu \mathrm{M})$ caused prolongation of 
the APD in SQT1 conditions at a basic cycle length (BCL) of $850 \mathrm{~ms}$, which roughly corresponds to $70 \mathrm{bpm}$ (Figure 3Ai). The prolongation in APD was found to be predominantly due to the blocking actions of DISO on $h E R G$ channel $I_{\mathrm{Kr}}$ currents (Figure 3Aii), with minimal changes in the APD resulting from block of other currents (not shown).

At a concentration of $10 \mu \mathrm{M}$, the fractional block due to DISO on affected ionic currents was as follows (in descending order): WT $I_{\text {KrhERG }}(48.3 \%)$, N588K $I_{\text {KrhERG }}$ $(42.9 \%), I_{\mathrm{Na}}(4.4 \%), I_{\mathrm{to}}(3.0 \%)$, and $I_{\mathrm{CaL}}(1.0 \%)$ Changes to the $\mathrm{APD}_{90}$ and peak $I_{\mathrm{Kr}}$ current density due to varying concentrations of DISO are summarised in Figure 3B, where it can be seen that both changed in a concentrationdependent manner.
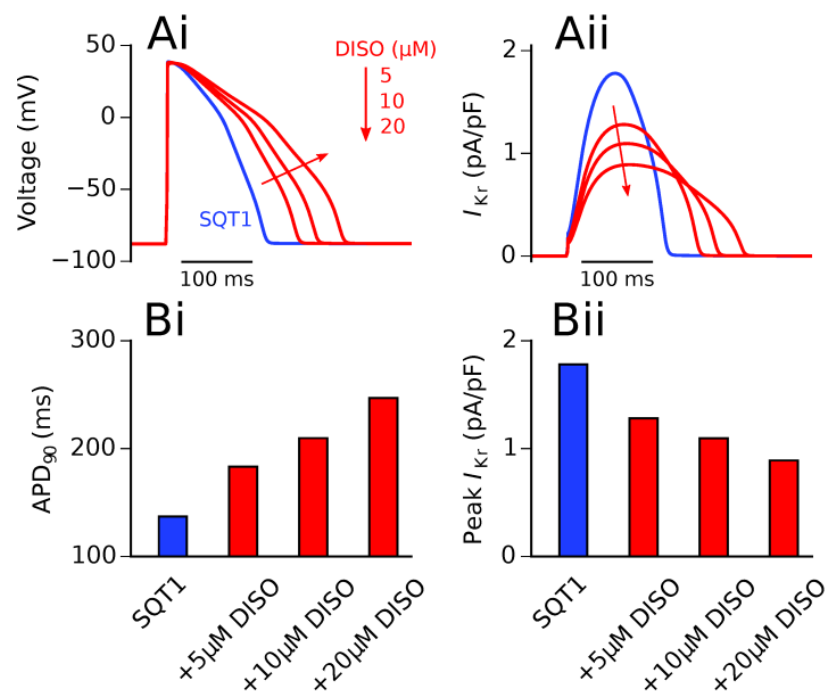

Figure 3: Effects of varying concentrations of DISO (5 $\mu \mathrm{M}, 10 \mu \mathrm{M}, 20 \mu \mathrm{M}$ ) on human ventricular endocardial (ENDO) AP waveforms and $\mathrm{APD}_{90}$ (Ai,Aii), and $I_{\mathrm{Kr}}$ current profile/peak density (Bi,Bii) in SQT1 conditions. The drug-free SQT1 AP and characteristics are shown in blue. $\mathrm{BCL}=850 \mathrm{~ms}$.

\subsection{D simulations}

The 1D transmural ventricular strand model was also paced at a BCL of $850 \mathrm{~ms}$, using the aforementioned protocol. Space-time plots of AP propagation in the 1D strand are shown in Figure 4. The distribution of the membrane potential across the 1D transmural strand is shown using a standard rainbow palette, ranging from -85 to $+35 \mathrm{mV}$. The pseudo-ECG was computed in SQT1 conditions, and under application of $10 \mu \mathrm{M}$ DISO. A concentration of $10 \mu \mathrm{M}$ DISO prolonged the corrected QT interval from $263 \mathrm{~ms}$ to $338 \mathrm{~ms}$.

\section{Discussion}

A biophysically-detailed Markov chain formulation of the SQT1 phenotype [11] has been incorporated into an established model of the human ventricular action potential incorporating transmural heterogeneities [10]. The resulting model has been used to investigate the functional consequences of the class 1a anti-arrhythmic agent disopyramide (DISO) on drug-induced APD and QT interval prolongation.

The therapeutic steady-state plasma levels of DISO are reported to be $2.8-4.4 \mathrm{mg} / \mathrm{L}$ [17], which corresponds to a dose of $\sim 8.3-13.0 \mu \mathrm{M}$. Several doses $(5 \mu \mathrm{M}, 10 \mu \mathrm{M}, 20$ $\mu \mathrm{M})$ were simulated in order to cover a range of possibilities, including low and high doses.

Our simulation results showed that combined blocking effects of DISO on $I_{\mathrm{Kr}}, I_{\mathrm{Na}}, I_{\mathrm{CaL}}$, and $I_{\mathrm{to}}$ caused significant prolongation of the action potential duration (APD). At a clinically-relevant concentration the $I_{\mathrm{Kr}}$ current was affected the most (even factoring in reduced blocking potency in SQT1-related N588K mutant hERG channels), with a smaller degree of $I_{\mathrm{Na}} / I_{\mathrm{to}}$ block and almost negligible block of $I_{\mathrm{CaL}}$. In the $1 \mathrm{D}$ transmural ventricular strand model, a concentration of $10 \mu \mathrm{M}$ produced a significant prolongation of the QT interval, bringing it much closer to the normal range of QT intervals. This is consistent with findings from the pilot study of Schimpf et al. [9], in which oral DISO successfully prolonged the QT interval and ventricular ERP in vivo.

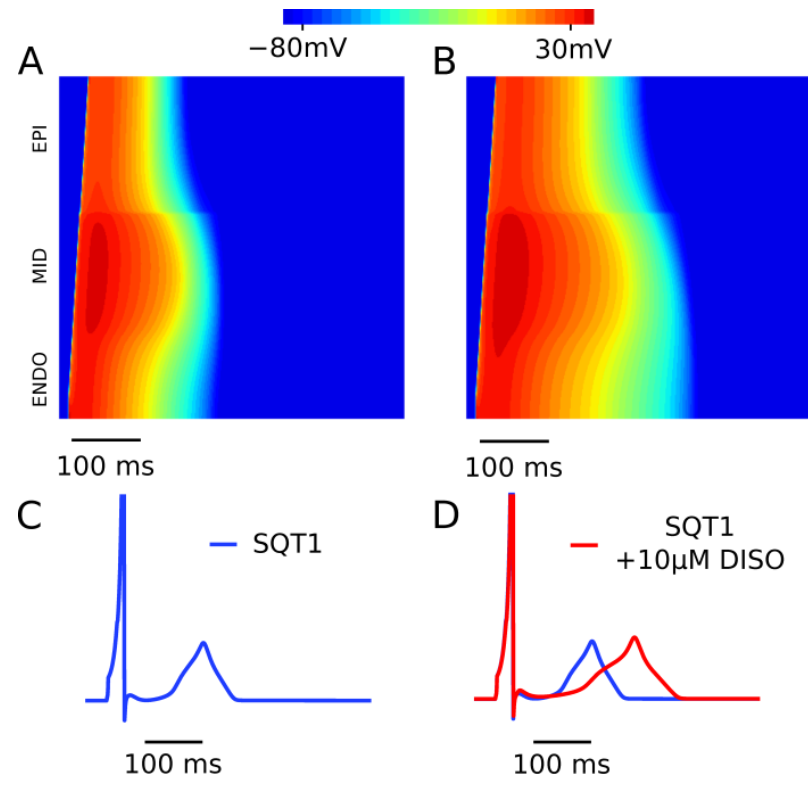

Figure 4: Dose-dependent effects of DISO on the pseudoECG of a 1D transmural strand of human ventricular tissue in SQT1 conditions (A), with corresponding QTc intervals (B) as calculated using Framingham's QT correction formula. $\mathrm{BCL}=850 \mathrm{~ms}$.

Reduced ERP due to N588K mutant $h E R G$ channels in the human ventricles was shown to be an important factor 
in increasing susceptibility to re-entrant arrhythmias in a study by Adeniran et al. [4]. The APD prolongation due to application of DISO is likely to partially/fully restore the ERP, de-stabilising re-entrant circuits in tissue and increasing likelihood of arrhythmia termination.

\section{Conclusion}

As the SQTS is very rare, extensive clinical trials are currently not feasible. However, computational modelling can be a powerful tool in assessing drug-induced APD/QT interval prolongation, as well as helping to dissect underlying ionic mechanisms. In the present study, we have demonstrated that the class 1a antiarrhythmic agent disopyramide is an effective inhibitor of mutant hERG channels associated with SQT1 in the human ventricles. This study provides new insights into a potential pharmacological option in the treatment of SQT1 and other $h E R G$-mediated forms of SQTS.

\section{Acknowledgements}

This project was funded by the British Heart Foundation (FS/14/5/30533).

\section{References}

[1] R. Schimpf, C. Wolpert, F. Gaita, C. Giustetto, and M. Borggrefe, "Short QT syndrome," Cardiovasc. Res., vol. 67, no. 3, pp. 357-366, Aug. 2005.

[2] R. Brugada, K. Hong, R. Dumaine, J. Cordeiro, F. Gaita, M. Borggrefe, T. M. Menendez, J. Brugada, G. D. Pollevick, C. Wolpert, E. Burashnikov, K. Matsuo, Y. S. Wu, A. Guerchicoff, F. Bianchi, C. Giustetto, R. Schimpf, P. Brugada, and C. Antzelevitch, "Sudden Death Associated With Short-QT Syndrome Linked to Mutations in HERG," Circulation, vol. 109, no. 1, pp. 30-35, Jan. 2004.

[3] M. J. McPate, R. S. Duncan, J. T. Milnes, H. J. Witchel, and J. C. Hancox, "The N588K-HERG $\mathrm{K}+$ channel mutation in the 'short QT syndrome': Mechanism of gainin-function determined at $37^{\circ} \mathrm{C}$,” Biochem. Biophys. Res. Commun., vol. 334, no. 2, pp. 441-449, Aug. 2005.

[4] I. Adeniran, M. J. McPate, H. J. Witchel, J. C. Hancox, and H. Zhang, "Increased Vulnerability of Human Ventricle to Re-entrant Excitation in hERG-linked Variant 1 Short QT Syndrome,” PLoS Comput. Biol., vol. 7, no. 12, Dec. 2011.

[5] C. Giustetto, F. D. Monte, C. Wolpert, M. Borggrefe, R. Schimpf, P. Sbragia, G. Leone, P. Maury, O. Anttonen, M. Haissaguerre, and F. Gaita, "Short QT syndrome: clinical findings and diagnostic-therapeutic implications," Eur. Heart J., vol. 27, no. 20, pp. 2440-2447, Oct. 2006.

[6] J. Villafañe, J. Atallah, M. H. Gollob, P. Maury, C. Wolpert, R. Gebauer, H. Watanabe, M. Horie, O. Anttonen, P. Kannankeril, B. Faulknier, J. Bleiz, T. Makiyama, W. Shimizu, R. M. Hamilton, and M.-L. Young, "Long-Term Follow-Up of a Pediatric Cohort With Short QT Syndrome,” J. Am. Coll. Cardiol., vol. 61, no.
11, pp. 1183-1191, Mar. 2013.

[7] M. J. McPate, R. S. Duncan, H. J. Witchel, and J. C. Hancox, "Disopyramide is an effective inhibitor of mutant HERG $\mathrm{K}+$ channels involved in variant 1 short QT syndrome,” J. Mol. Cell. Cardiol., vol. 41, no. 3, pp. 563566, Sep. 2006.

[8] C. Giustetto, R. Schimpf, A. Mazzanti, C. Scrocco, P. Maury, O. Anttonen, V. Probst, J.-J. Blanc, P. Sbragia, P. Dalmasso, M. Borggrefe, and F. Gaita, "Long-Term Follow-Up of Patients With Short QT Syndrome,” J. Am. Coll. Cardiol., vol. 58, no. 6, pp. 587-595, Aug. 2011.

[9] R. Schimpf, C. Veltmann, C. Giustetto, F. Gaita, M. Borggrefe, and C. Wolpert, "In vivo Effects of Mutant HERG K+ Channel Inhibition by Disopyramide in Patients with a Short QT-1 Syndrome: A Pilot Study," $J$. Cardiovasc. Electrophysiol., vol. 18, no. 11, pp. 11571160, Nov. 2007.

[10] T. O’Hara, L. Virág, A. Varró, and Y. Rudy, “Simulation of the Undiseased Human Cardiac Ventricular Action Potential: Model Formulation and Experimental Validation,” PLoS Comput. Biol., vol. 7, no. 5, May 2011.

[11] D. G. Whittaker, M. A. Colman, H. Ni, J. C. Hancox, and H. Zhang, "In silico investigation of short QT syndromelinked potassium channel mutations on electro-mechanical function of human atrial cells," in Computing in Cardiology Conference (CinC), 2015, 2015, pp. 853-856.

[12] N. Jost, L. Virág, P. Comtois, B. Ördög, V. Szuts, G. Seprényi, M. Bitay, Z. Kohajda, I. Koncz, N. Nagy, T. Szél, J. Magyar, M. Kovács, L. G. Puskás, C. Lengyel, E. Wettwer, U. Ravens, P. P. Nánási, J. G. Papp, A. Varró, and S. Nattel, "Ionic mechanisms limiting cardiac repolarization reserve in humans compared to dogs," $J$. Physiol., vol. 591, no. Pt 17, pp. 4189-4206, Sep. 2013.

[13] C. Yasuda, S. Yasuda, H. Yamashita, J. Okada, T. Hisada, and S. Sugiura, "The human ether-a-go-go-related gene (hERG) current inhibition selectively prolongs action potential of midmyocardial cells to augment transmural dispersion," J. Physiol. Pharmacol. Off. J. Pol. Physiol. Soc., vol. 66, no. 4, pp. 599-607, Aug. 2015.

[14] J. A. Sanchez-Chapula, "Mechanism of Transient Outward K+ Channel Block by Disopyramide,” J. Pharmacol. Exp. Ther., vol. 290, no. 2, pp. 515-523, Aug. 1999.

[15] T. Brennan, M. Fink, and B. Rodriguez, "Multiscale modelling of drug-induced effects on cardiac electrophysiological activity,” Eur. J. Pharm. Sci., vol. 36, no. 1, pp. 62-77, Jan. 2009.

[16] K. Gima and Y. Rudy, "Ionic Current Basis of Electrocardiographic Waveforms A Model Study,” Circ. Res., vol. 90, no. 8, pp. 889-896, May 2002.

[17] I. L. D. Nauta, J. van de Calseyde, and D. P. Hertzberger, "Plasma levels of disopyramide after administration of conventional capsules and sustained-release tablets," Curr. Med. Res. Opin., vol. 8, no. 8, pp. 582-593, Jan. 1983.

Address for correspondence.

Dominic G Whittaker.

3.08. Schuster Laboratory,

University of Manchester,

Manchester, UK,

M13 9PL,

dominic.whittaker@manchester.ac.uk 